Algebraic 83 Geometric $\mathcal{T}$ opology

Volume 5 (2005) 219-235

Published: 6 April 2005

ATG

\title{
Rational acyclic resolutions
}

\author{
Michael Levin
}

\begin{abstract}
Let $X$ be a compactum such that $\operatorname{dim}_{\mathbb{Q}} X \leq n, n \geq 2$. We prove that there is a $\mathbb{Q}$-acyclic resolution $r: Z \longrightarrow X$ from a compactum $Z$ of $\operatorname{dim} \leq n$. This allows us to give a complete description of all the cases when for a compactum $X$ and an abelian group $G$ such that $\operatorname{dim}_{G} X \leq n$, $n \geq 2$ there is a $G$-acyclic resolution $r: Z \longrightarrow X$ from a compactum $Z$ of $\operatorname{dim} \leq n$.
\end{abstract}

AMS Classification 55M10, 54F45

Keywords Cohomological dimension, acyclic resolution

\section{Introduction}

The cohomological dimension $\operatorname{dim}_{G} X$ of a separable metric space $X$ with respect to an abelian group $G$ is the least number $n$ such that $\check{H}^{n+1}(X, A ; G)=0$ for every closed subset $A$ of $X$. It was known long ago that $\operatorname{dim} X=\operatorname{dim}_{\mathbb{Z}} X$ if $X$ is finite dimensional. Solving an outstanding problem in cohomological dimension theory Dranishnikov constructed in 1987 an infinite dimensional compactum (= compact metric space) with $\operatorname{dim}_{\mathbb{Z}}=3$. A few years earlier a deep relation between $\operatorname{dim}_{\mathbb{Z}}$ and $\operatorname{dim}$ was established by the Edwards cell-like resolution theorem [1, 10] saying that a compactum of $\operatorname{dim}_{\mathbb{Z}} \leq n$ can be obtained as the image of a cell-like map defined on a compactum of $\operatorname{dim} \leq n$. A compactum $X$ is cell-like if any map $f: X \longrightarrow K$ from $X$ to a CW-complex $K$ is null homotopic. A map is cell-like if its fibers are cell-like. The reduced Čech cohomology groups of a cell-like compactum are trivial with respect to any group $G$.

Acyclic resolutions are a natural generalization of the Edwards cell-like resolution. A space is $G$-acyclic if its reduced Čech cohomology groups modulo $G$ are trivial, a map is $G$-acyclic if every fiber is $G$-acyclic. As a consequence of the Vietoris-Begle theorem a surjective $G$-acyclic map of compacta cannot raise the cohomological dimension $\operatorname{dim}_{G}$. 
Let $\mathcal{P}$ denote the set of prime numbers and let $\mathcal{L} \subset \mathcal{P}$. Recall that

$\mathbb{Z}_{(\mathcal{L})}=\{m / n: m \in \mathbb{Z}, n \in \mathbb{N}$ and $n$ is not divisible by the elements of $\mathcal{L}\}$.

Thus $\mathbb{Z}_{(\emptyset)}=\mathbb{Q}$ and $\mathbb{Z}_{(\mathcal{P})}=\mathbb{Z}$. Dranishnikov [4, 5] proved the following:

Theorem 1.1 [4, 5] Let $\mathcal{L} \subset \mathcal{P}$ and let $X$ be a compactum with $\operatorname{dim}_{\mathbb{Z}_{(\mathcal{L})}} X \leq$ $n, n \geq 2$. Then there are a compactum $Z$ with $\operatorname{dim} Z \leq n+1$ and $\operatorname{dim}_{\mathbb{Z}_{(\mathcal{L})}} Z \leq$ $n$ and a $\mathbb{Z}_{(\mathcal{L})}$-acyclic map $r: Z \longrightarrow X$ from $Z$ onto $X$.

In [7. Theorem 1.1 was generalized for arbitrary groups.

Theorem 1.2 [7] Let $G$ be an abelian group and let $X$ be a compactum with $\operatorname{dim}_{G} X \leq n, n \geq 2$. Then there are a compactum $Z$ with $\operatorname{dim} Z \leq n+1$ and $\operatorname{dim}_{G} Z \leq n$ and a $G$-acyclic map $r: Z \longrightarrow X$ from $Z$ onto $X$.

It is known that in general in Theorem 1.2 the dimension of $Z$ cannot be reduced to $n$ [6]. However, Dranishnikov [5] showed that it can be done for $G=\mathbb{Z}_{p}$.

Theorem 1.3 ([5], cf. [8]) Let $\mathcal{L} \subset \mathcal{P}$ and let $X$ be a compactum with $\operatorname{dim}_{\mathbb{Z}_{p}} X \leq n$ for every $p \in \mathcal{L}$. Then there are a compactum $Z$ with $\operatorname{dim} Z \leq n$ and a map $r: Z \longrightarrow X$ from $Z$ onto $X$ such that $r$ is $\mathbb{Z}_{p}$-acyclic for every $p \in \mathcal{L}$.

One of the key problems in the area of acyclic resolutions is whether the dimension of $Z$ in Theorem 1.1 can be reduced to $n$. The main purpose of this paper is to answer this problem affirmatively by proving:

Theorem 1.4 Let $\mathcal{L} \subset \mathcal{P}$ and let $X$ be a compactum with $\operatorname{dim}_{\mathbb{Z}_{(\mathcal{L})}} X \leq n$, $n \geq 2$. Then there are a compactum $Z$ with $\operatorname{dim} Z \leq n$ and a $\mathbb{Z}_{(\mathcal{L}) \text {-acyclic }}$ map $r: Z \longrightarrow X$ from $Z$ onto $X$.

Theorems 1.3 and 1.4 allow us to give a complete description of all the cases when for an abelian group $G$ and a compactum $X$ such that $\operatorname{dim}_{G} X \leq n$, $n \geq 2$ there is a $G$-acyclic resolution $r: Z \longrightarrow X$ from a compactum $Z$ of $\operatorname{dim} \leq n$.

Recall that the Bockstein basis of abelian groups is the following collection of groups $\sigma=\left\{\mathbb{Q}, \mathbb{Z}_{p}, \mathbb{Z}_{p^{\infty}}, \mathbb{Z}_{(p)}: p \in \mathcal{P}\right\}$. The Bockstein basis $\sigma(G)$ of an abelian group $G$ is a subcollection of $\sigma$ defined as follows: 


$$
\begin{aligned}
& \mathbb{Z}_{(p)} \in \sigma(G) \text { if } G / \operatorname{Tor} G \text { is not divisible by } p \\
& \mathbb{Z}_{p} \in \sigma(G) \text { if } \operatorname{Tor}_{p} G \text { is not divisible by } p ; \\
& \mathbb{Z}_{p^{\infty}} \in \sigma(G) \text { if } \operatorname{Tor}_{p} G \neq 0 \text { and } \operatorname{Tor}_{p} G \text { is divisible by } p ; \\
& \mathbb{Q} \in \sigma(G) \text { if } G / \operatorname{Tor} G \neq 0 \text { and } G / \operatorname{Tor} G \text { is divisible by every } p \in \mathcal{P} .
\end{aligned}
$$

Let $X$ be a compactum. The Bockstein theorem says that

$$
\operatorname{dim}_{G} X=\sup \left\{\operatorname{dim}_{E} X: E \in \sigma(G)\right\} .
$$

It is known [6] that $X$ is $G$-acyclic if and only if $X$ is $E$-acyclic for every $E \in \sigma(G)$.

Following 9] define the closure $\overline{\sigma(G)}$ of the Bockstein basis $\sigma(G)$ of $G$ as a collection of abelian groups containing $\sigma(G)$ and possibly some additional groups determined by:

$$
\begin{aligned}
& \mathbb{Z}_{p} \in \overline{\sigma(G)} \text { if } \mathbb{Z}_{p^{\infty}} \in \overline{\sigma(G)} ; \\
& \mathbb{Z}_{p^{\infty}} \in \overline{\sigma(G)} \text { if } \mathbb{Z}_{p} \in \overline{\sigma(G)} ; \\
& \mathbb{Q} \in \overline{\sigma(G)} \text { if } \mathbb{Z}_{(p)} \in \overline{\sigma(G)} ; \\
& \mathbb{Z}_{(p)} \in \overline{\sigma(G)} \text { if } \mathbb{Q} \text { and } \mathbb{Z}_{p^{\infty}} \in \overline{\sigma(G)} .
\end{aligned}
$$

Theorem 1.5 Let $G$ be an abelian group, let $X$ be a compactum and let $n \geq 2$. Then there exist a compactum $Z$ of $\operatorname{dim} \leq n$ and a $G$-acyclic map $r: Z \longrightarrow X$ from $Z$ onto $X$ if and only if $\operatorname{dim}_{E} X \leq n$ for every $E \in \overline{\sigma(G)}$.

Proof Assume that there exist a compactum $Z$ of $\operatorname{dim} \leq n$ and a $G$-acyclic map $r: Z \longrightarrow X$ from $Z$ onto $X$. Then $r$ is $E$-acyclic for every $E \in \sigma(G)$. Consider the exact sequences:

$$
\begin{aligned}
& 0 \longrightarrow \mathbb{Z}_{p} \longrightarrow \mathbb{Z}_{p^{\infty}} \longrightarrow \mathbb{Z}_{p^{\infty}} \longrightarrow 0 ; \\
& 0 \longrightarrow \mathbb{Z}_{p^{k}} \longrightarrow \mathbb{Z}_{p^{k+1}} \longrightarrow \mathbb{Z}_{p} \longrightarrow 0 ; \\
& 0 \longrightarrow \mathbb{Z}_{(p)} \longrightarrow \mathbb{Z}_{(p)} \longrightarrow \mathbb{Z}_{p} \longrightarrow 0 ; \\
& 0 \longrightarrow \mathbb{Z}_{(p)} \longrightarrow \mathbb{Q} \longrightarrow \mathbb{Z}_{p^{\infty}} \longrightarrow 0
\end{aligned}
$$

Then it follows from the Bockstein sequences generated by the above exact sequences that in the class of compacta:

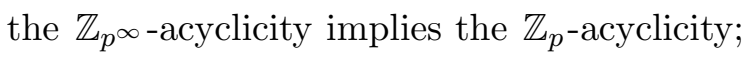

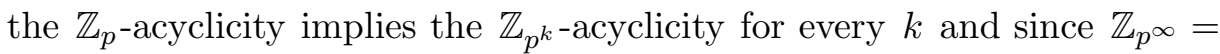

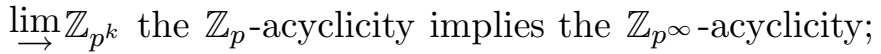


the $\mathbb{Z}_{(p)}$-acyclicity implies the $\mathbb{Z}_{p \text {-acyclicity; }}$

the $\mathbb{Z}_{(p)}$-acyclicity implies the $\mathbb{Q}$-acyclicity;

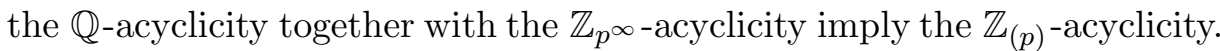

From these properties we obtain that $r$ is $E$-acyclic for every $E \in \overline{\sigma(G)}$ and therefore by the Vietoris-Begle theorem $\operatorname{dim}_{E} X \leq n$ for every $E \in \overline{\sigma(G)}$.

Now assume that $\operatorname{dim}_{E} X \leq n$ for every $E \in \overline{\sigma(G)}$.

Consider first the case when $G$ is torsion. Let $\mathcal{L}=\left\{p \in \mathcal{P}: \operatorname{Tor}_{p} G \neq 0\right\}$. Then $\operatorname{dim}_{\mathbb{Z}_{p}} X \leq n$ for every $p \in \mathcal{L}$ and the existence of the required resolution follows from Theorem 1.3 and the above properties of acyclicity.

Assume that $G$ is not torsion. Then $\mathbb{Q} \in \overline{\sigma(G)}$. Define $\mathcal{L}=\left\{p \in \mathcal{P}: \mathbb{Z}_{(p)} \in\right.$ $\overline{\sigma(G)}\}$. By the Bockstein theorem $\operatorname{dim}_{\mathbb{Z}_{(\mathcal{L})}} X \leq n$ and therefore by Theorem 1.4 there exists a $\mathbb{Z}_{(\mathcal{L})}$-acyclic resolution $r: Z \longrightarrow X$ from a compactum $Z$ of $\operatorname{dim} \leq n$ onto $X$. Clearly $r$ is $\mathbb{Q}$-acyclic if $\mathcal{L}=\emptyset$. If $\mathcal{L} \neq \emptyset$ then $r$ is

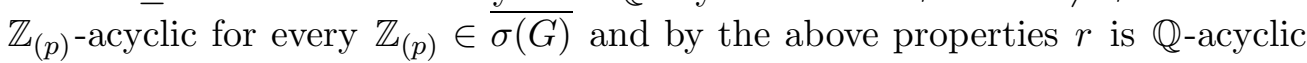
as well.

Let $\mathbb{Z}_{(p)} \in \sigma(G)$. Then $\mathbb{Z}_{(p)} \in \overline{\sigma(G)}$ and therefore $r$ is $\mathbb{Z}_{(p)}$-acyclic.

Let $\mathbb{Z}_{p}$ or $\mathbb{Z}_{p^{\infty}} \in \sigma(G)$. Then $\mathbb{Z}_{p^{\infty}} \in \overline{\sigma(G)}$ and since $\mathbb{Q} \in \overline{\sigma(G)}$ we have that $\mathbb{Z}_{(p)} \in \overline{\sigma(G)}$. Hence $r$ is $\mathbb{Z}_{(p)}$-acyclic and by the above properties $r$ is also $\mathbb{Z}_{p}$-acyclic and $\mathbb{Z}_{p^{\infty} \text {-acyclic. }}$

Thus we have shown that $r$ is $E$-acyclic for every $E \in \sigma(G)$ and hence $r$ is $G$-acyclic. The theorem is proved.

Theorem 1.2 was generalized in $[9]$ as follows.

Theorem 1.6 [9] Let $X$ be a compactum. Then for every integer $n \geq 2$ there are a compactum $Z$ of $\operatorname{dim} \leq n+1$ and a surjective map $r: Z \longrightarrow X$ having the property that for every abelian group $G$ and every integer $k \geq 2$ such that $\operatorname{dim}_{G} X \leq k \leq n$ we have that $\operatorname{dim}_{G} Z \leq k$ and $r$ is $G$-acyclic.

Theorem [1.6] resulted in the following conjecture posed in 9].

Conjecture 1.7 [9] Let $\mathcal{G}$ be a collection of abelian groups, let $X$ be a compactum $X$ and let $n \geq 2$. Then there exist a compactum $Z$ of $\operatorname{dim} \leq n$ and a surjective map $r: Z \longrightarrow X$ such that $\operatorname{dim}_{G} Z \leq \max \left\{\operatorname{dim}_{G} X, 2\right\}$ and $r$ is $G$-acyclic for every $G \in \mathcal{G}$ if and only if $\operatorname{dim}_{E} X \leq n$ for every $E \in \overline{\sigma(\mathcal{G})}=$ $\cup\{\overline{\sigma(G)}: G \in \mathcal{G}\}$. 
It is easy to derive from the proof of Theorem 1.5 that the condition $\operatorname{dim}_{E} X \leq n$ for every $E \in \overline{\sigma(\mathcal{G})}$ is necessary for the existence of a resolution required in the conjecture. Theorem 1.5 seems to be an important step toward proving that this condition is also sufficient.

Note that neither the restriction $k \geq 2$ in Theorem [1.6] nor the restriction $\operatorname{dim}_{G} Z \leq \max \left\{\operatorname{dim}_{G} X, 2\right\}$ in Conjecture 1.7 can be replaced by $k \geq 1$ and $\operatorname{dim}_{G} Z \leq \operatorname{dim}_{G} X$ respectively.

Indeed, let a compactum $Y$ be $\mathbb{Q}$-acyclic and $\operatorname{dim}_{\mathbb{Q}} Y \leq 1$. Then for every closed subset $A$ of $Y$ the exact sequence of the pair $(Y, A)$ implies that $\check{H}^{1}(A ; \mathbb{Q})$ $=0$ and by Universal Coefficient Theorem $\check{H}^{1}(A)=0$. Hence $\check{H}^{1}(Y)=0$ and $\operatorname{dim} Y \leq 1$ since every map from $A$ to $\mathbb{S}^{1}=K(\mathbb{Z}, 1)$ is null-homotopic and therefore extends over $Y$. Then $Y$ is $\mathbb{Z}$-acyclic. Thus we obtain that a $\mathbb{Q}$ acyclic map from a compactum of $\operatorname{dim}_{\mathbb{Q}} \leq 1$ is $\mathbb{Z}$-acyclic and hence there is no $\mathbb{Q}$-acyclic resolution $r: Z \longrightarrow X$ for a compactum $X$ of $\operatorname{dim}_{\mathbb{Z}}=\infty$ and $\operatorname{dim}_{\mathbb{Q}}=1$ from a finite dimensional compactum $Z$ of $\operatorname{dim}_{\mathbb{Q}} \leq 1$.

The last observation also shows that the restriction $n \geq 2$ in Theorem 1.4 cannot be omitted.

\section{Preliminaries}

The extensional dimension of a compactum $X$ is said not to exceed a CWcomplex $K$, written $\mathrm{e}-\operatorname{dim} X \leq K$, if for every closed subset $A$ of $X$ and every map $f: A \longrightarrow K$ there is an extension of $f$ over $X$. It is well-known that $\operatorname{dim} X \leq n$ is equivalent to e $-\operatorname{dim} X \leq \mathbb{S}^{n}$ and $\operatorname{dim}_{G} X \leq n$ is equivalent to $\mathrm{e}-\operatorname{dim} X \leq K(G, n)$, where $K(G, n)$ is an Eilenberg-Mac Lane complex of type $(G, n)$. Also note that if $\operatorname{dim} X \leq n+1, n \geq 2$ then $\operatorname{dim}_{G} X \leq n$ is equivalent to $\mathrm{e}-\operatorname{dim} X \leq M(G, n)$, where $M(G, n)$ is a simply-connected Moore complex of type $(G, n)$ (the condition $\operatorname{dim} X \leq n+1$ can be relaxed to $\operatorname{dim} X<\infty$ [3] $)$.

A map between $\mathrm{CW}$-complexes is said to be combinatorial if the preimage of every subcomplex of the range is a subcomplex of the domain.

Let $M$ be a CW-complex. We say that a triangulation of $M$ is compatible with the $\mathrm{CW}$-structure if every cell is a simplicial subcomplex.

Let $\mathcal{L} \subset \mathcal{P}$. We will call a simplicial complex $M$ an $n$-dimensional $\mathcal{L}$-sphere if the following conditions are satisfied:

$M$ is a finite $n$-dimensional simplicial complex; 
$\tilde{H}_{i}\left(M ; \mathbb{Z}_{(\mathcal{L})}\right)=0$ if $i \neq n$ and $\tilde{H}_{n}\left(M ; \mathbb{Z}_{(\mathcal{L})}\right)=\mathbb{Z}_{(\mathcal{L})} ;$

for every $n$-simplex $\Delta$ of $M$, the subcomplex $B=(M \backslash \Delta) \cup \partial \Delta$ is $\mathbb{Z}_{(\mathcal{L})^{-}}$ acyclic. (Note that by Universal Coefficient Theorem the $\mathbb{Z}_{(\mathcal{L})}$-acyclicity of $B$ is equivalent to $\tilde{H}_{*}\left(B ; \mathbb{Z}_{(\mathcal{L})}\right)=0$.)

It is easy to verify that if $M$ is an $n$-dimensional $\mathcal{L}$-sphere with respect to a given triangulation then $M$ is also an $n$-dimensional $\mathcal{L}$-sphere with respect to any barycentric subdivision of the triangulation.

By an $n$-dimensional $\mathcal{L}$-ball $B$ we mean an $n$-dimensional $\mathcal{L}$-sphere without the interior of one of its $n$-simplexes. The boundary of the simplex whose interior is removed is said to be the boundary of the $\mathcal{L}$-ball $B$ and it is denoted by $\partial B$.

Saying that an $n$-dimensional simplicial complex $N$ is obtained from an $n$ dimensional simplicial complex $M$ by replacing an $n$-simplex $\Delta$ of $M$ by an $n$-dimensional $\mathcal{L}$-ball $B$ we mean that the interior of $\Delta$ is removed from $M$ and $B$ is attached to the boundary of $\Delta$ by a simplicial homeomorphism of $\partial \Delta$ and $\partial B$. We regard $N$ as a simplicial complex with respect to the natural triangulation induced by the triangulations of $M$ and $B$.

It is clear that replacing an $n$-simplex of an $n$-dimensional $\mathcal{L}$-sphere by an $n$ dimensional $\mathcal{L}$-ball we obtain again an $n$-dimensional $\mathcal{L}$-sphere and replacing an $n$-simplex of an $n$-dimensional $\mathcal{L}$-ball by an $n$-dimensional $\mathcal{L}$-ball we obtain again an $n$-dimensional $\mathcal{L}$-ball.

Proposition 2.1 Let $\mathcal{L} \subset \mathcal{P}$, let $X$ be an compactum with $\operatorname{dim}_{\mathbb{Z}_{(\mathcal{L})}} X \leq n$ and $\operatorname{dim} X \leq n+1, n \geq 2$ and let $f: X \longrightarrow K$ be a map from $X$ to an $(n+1)$-dimensional finite simplicial complex $K$. Then for every $\epsilon>0$ there exist an $(n+1)$-dimensional finite $C W$-complex $M$ and maps $\phi: X \longrightarrow M$, $\psi: M \longrightarrow K$ such that:

(i) $f$ and $\psi \circ \phi$ are $\epsilon$-close, that is $\rho(f(x), \psi(\phi(x)))<\epsilon$ for every $x \in X$ where $\rho$ is a metric in $K$;

(ii) $\psi$ is combinatorial; $\psi$ is 1-to-1 over every simplex of $K$ which is not contained in an $(n+1)$-simplex of $K$; the $(n+1)$-cells of $M$ are exactly the preimages of the $(n+1)$-simplexes of $K$ under $\psi$ such that the interior points of the $(n+1)$-cells of $M$ are sent to the interior of the corresponding $(n+1)$ simplexes of $K$;

(iii) each $(n+1)$-cell $C$ of $M$ has a point $b \in \partial C$ such that $b$ is sent by $\psi$ to the interior of the corresponding $(n+1)$-simplex of $K$. We will call $b$ a free 
boundary point of $C$. It is clear from (ii) that $C$ is the only $(n+1)$-cell of $M$ containing $b$;

(iv) $M$ admits a compatible triangulation with respect to which the boundary of every $(n+1)$-cell of $M$ is an $n$-dimensional $\mathcal{L}$-sphere. Moreover: by (iii) such a triangulation of $M$ can be chosen such that for each cell $C$ of $M$ there is an $n$-simplex contained in $\partial C$ and consiting only of free boundary points of $C$.

Proof Fix an $n$-simplex $\Delta$ of $K$ contained in at least one $(n+1)$-simplex and let $\Delta_{1}, \Delta_{2}, \ldots, \Delta_{k}, k \geq 1$ be the $(n+1)$-simplexes of $L$ containing $\Delta$. Take a small closed $n$-dimensional ball $B$ contained in $\Delta$ such that $B$ does not touch the boundary of $\Delta$ and $B$ is centered at the barycenter $c$ of $\Delta$. For every $i=1,2, . ., k$ take a point $p_{i}$ sufficiently close to $B$ and contained in the interior of $\Delta_{i}$. Denote $P=\left\{p_{1}, \ldots, p_{k}\right\}$. Consider the join $B * p_{i}$ as the subset of $\Delta_{i}$ which is the cone over $B$ with the vertex at $p_{i}$. Thus we regard $B * P=\cup B * p_{i}$ as a subset of $\cup \Delta_{i}$. Let the $(n-1)$-dimensional sphere $S$ be the boundary $\partial B$ of $B$. Denote $F=f^{-1}(B * P)$ and $A=f^{-1}(S * P)$.

Represent a Moore space of type $\left(\mathbb{Z}_{(\mathcal{L})}, n-1\right)$ as an infinite telescope $T$ of a sequence of $(n-1)$-dimensional spheres with bonding maps of non-zero degrees which are not divisible by the elements of $\mathcal{L}$. By a finite subtelescope of $T$ we mean a subspace of $T$ which is the telescope of finitely many consecutive spheres in the sequence. Define the group $G$ as $G=0$ if $k=1$ and $G=$ the direct sum $\oplus \mathbb{Z}_{(\mathcal{L})}$ of $k-1$ copies of $\mathbb{Z}_{(\mathcal{L})}$ if $k \geq 2$. Let $D=\left\{d_{1}, d_{2}, \ldots, d_{k}\right\}$ be a discrete space of $k$ singletons. Then $T * D$ is a simply connected Moore space of type $(G, n)$. Let $\tau: S \longrightarrow T$ be an embedding of $S$ as the first sphere of the telescope $T$ and let $\delta: P \longrightarrow D$ be the map sending $p_{i}$ to $d_{i}$.

Consider the map $\alpha=\left.(\tau * \delta) \circ f\right|_{\ldots}: A \longrightarrow T * D$. By Bockstein Theorem $\operatorname{dim}_{G} X \leq n$ and since $\operatorname{dim} X \leq n+1$ we have that e $-\operatorname{dim} X \leq T * D$. Then $\alpha: A \longrightarrow T * D$ extends to $\beta: F \longrightarrow T * D$. Since $\beta(F)$ is compact take a finite subtelescope $T_{\beta}$ of $T$ starting at the first sphere and ending at a sphere $S_{\beta}$ such that $\beta(F)$ is contained in $T_{\beta} * D$ and let $\gamma: T_{\beta} \longrightarrow S_{\beta}$ be the natural retraction sending $T_{\beta}$ to the last sphere of $T_{\beta}$.

Define a CW-complex $M_{\Delta}$ as the quotient space of $L=(K \backslash(B * P)) \cup(S * P)$ obtained by identifying the points of $S * P$ with $S_{\beta} * D$ according to the map $(\gamma \circ \tau) * \delta: S * P \longrightarrow S_{\beta} * D$. Denote by $\pi: L \longrightarrow M_{\Delta}$ the projection and consider $S_{\beta} * D$ as the subset $\pi(S * P)$ of $M_{\Delta}$. We will also consider $L \backslash(B * P)$ as a subset of $M_{\Delta}$. Note that since the identifications on $S_{\beta} * D$ are induced 
by the join $(\gamma \circ \tau) * \delta$ we have that $\pi^{-1}\left(\pi\left(S * p_{i}\right)\right)=\pi^{-1}\left(S_{\beta} * d_{i}\right)=S * p_{i}$, $\pi^{-1}\left(\pi\left(p_{i}\right)\right)=\pi^{-1}\left(d_{i}\right)=p_{i}$ and $\pi^{-1}(\pi(S))=\pi^{-1}\left(S_{\beta}\right)=S$.

It is easy to see that $\left(\gamma * i d_{D}\right)(\alpha(x))=\pi(f(x)) \in S_{\beta} * D$ for $x \in A$ where $i d_{D}: D \longrightarrow D$ is the identity map. Then the map $\left.\pi \circ f\right|_{\ldots}:(X \backslash F) \cup A \longrightarrow M_{\Delta}$ extends to the map $\phi_{\Delta}: X \longrightarrow M_{\Delta}$ defined by $\phi_{\Delta}(x)=\left(\gamma * i d_{D}\right)(\beta(x))$ for $x \in F$.

Define a map $\psi_{\Delta}: M_{\Delta} \longrightarrow K$ such that:

every simplex of $K$ not intersecting $B * P$ and considered as a subset of $M_{\Delta}$ is sent by $\psi_{\Delta}$ to itself by the identity map;

$\psi_{\Delta}$ sends $\pi(\Delta \cap L)$ onto $\Delta$ such that $\pi(S)=S_{\beta}$ is sent to the barycenter $c$ of $\Delta$;

$\psi_{\Delta}$ sends $\pi\left(\Delta_{i} \cap L\right)$ onto $\Delta_{i}$ such that $\pi\left(\left(\Delta_{i} \backslash \partial \Delta_{i}\right) \cap L\right)$ is sent into $\Delta_{i} \backslash \partial \Delta_{i}$;

$\psi_{\Delta} \circ \phi$ is sufficiently close to $f$ provided that the ball $B$ and the points $p_{i} \in \Delta_{i}$ were chosen to be sufficiently close to the barycenter $c$ of $\Delta$.

Then for every $\Delta_{i}, \psi_{\Delta}^{-1}\left(\Delta_{i}\right)=\pi\left(\Delta_{i} \cap K\right)$ and since $\Delta_{i} \cap K=\left(\Delta_{i} \backslash\left(B * p_{i}\right)\right) \cup$ $\left(S * p_{i}\right)$ is homeomorphic to an $(n+1)$-dimensional Euclidean ball in which $\pi$ makes identifications only on the boundary we may endow $M_{\Delta}$ with a CWstructure so that $\psi_{\Delta}$ becomes a combinatorial map and the $(n+1)$-cells of $M_{\Delta}$ are exactly the preimages of the $(n+1)$-simplexes of $K$. Moreover, since the identifications on $S * p_{i}$ are induced by the map $\gamma: S \longrightarrow S_{\beta}$ of degree not divisible by the elements of $\mathcal{L}$ one can define a compatible triangulation of $M_{\Delta}$ so that the boundary of each cell $C_{i}=\psi_{\Delta}^{-1}\left(\Delta_{i}\right)$ becomes an $\mathcal{L}$-sphere. Finally note that $\pi\left(p_{i}\right)$ is a free boundary point of the cell $C_{i}$ and if a triangulation of $M_{i}$ is chosen to be sufficiently small then for every $i$ there will be an $n$-simplex contained in $\partial C_{i}$ consisting of free boundary points of $C_{i}$.

It is easy to see that the procedure of constructing $M_{\Delta}$ and $\psi_{\Delta}: M_{\Delta} \longrightarrow K$ described above can be carried out independently for all $n$-simplexes $\Delta$ of $L$ contained in some $(n+1)$-simplexes of $K$. This way we can construct a CW-complex $M$ and a map $\psi: M \longrightarrow K$ satisfying the conclusions of the proposition.

Let $K^{\prime}$ be a simplicial complex and let $\kappa: K \longrightarrow K^{\prime}, \lambda: L \longrightarrow L^{\prime}, \alpha: L \longrightarrow K$ and $\alpha^{\prime}: L^{\prime} \longrightarrow K^{\prime}$ be maps.

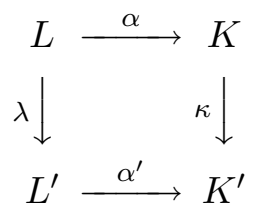

Algebraic 83 Geometric Topology, Volume 5 (2005) 
We say that $\kappa, \lambda, \alpha$ and $\alpha^{\prime}$ combinatorially commute if for every simplex $\Delta$ of $K^{\prime}$ we have that $\left(\alpha^{\prime} \circ \lambda\right)\left((\kappa \circ \alpha)^{-1}(\Delta)\right) \subset \Delta$. (The direction in which we want the maps $\kappa, \lambda, \alpha$ and $\alpha^{\prime}$ to combinatorially commute is indicated by the first map in the list. Thus saying that $\alpha^{\prime}, \kappa, \lambda$ and $\alpha$ combinatorially commute we would mean that $(\kappa \circ \alpha)\left(\left(\alpha^{\prime} \circ \lambda\right)^{-1}(\Delta)\right) \subset \Delta$ for every simplex $\Delta$ of $K^{\prime}$.)

Let $K$ and $K^{\prime}$ be simplicial complexes and let $\kappa: K \longrightarrow K^{\prime} \kappa^{\prime}: K \longrightarrow K^{\prime}$. We say that $\kappa^{\prime}$ is a simplicial approximation of $\kappa$ if $\kappa^{\prime}$ is a simplicial map and for every simplex $\Delta$ of $K^{\prime}, \kappa^{\prime}\left(\kappa^{-1}(\Delta)\right) \subset \Delta$. Thus if $\kappa^{\prime}$ is a simplicial approximation of $\kappa$ then for every subcomplex $N$ of $K^{\prime}$ and for every $A \subset K$ such that $\kappa(A) \subset N$ we also have $\kappa^{\prime}(A) \subset N$.

For a simplicial complex $K$ and $a \in K, \operatorname{st}(a)$ (the star of $a$ ) denotes the union of all the simplexes of $K$ containing $a$.

The following proposition whose proof is left to the reader is a collection of simple combinatorial properties of maps.

\section{Proposition 2.2}

(i) Let $\kappa: K \longrightarrow K^{\prime}$ be a combinatorial map of simplicial complexes $K$ and

$K^{\prime}$. Then $\kappa$ admits a simplicial approximation.

(ii) Let $\kappa: K \longrightarrow K^{\prime}$ be a map of simplicial complexes $K$ and $K^{\prime}$. Then there is a sufficiently small barycentric subdivision of the triangulation of $K$ with respect to which $\kappa$ admits a simplicial approximation.

(iii) Let $K$ and $K^{\prime}$ be simplicial complexes, let maps $\kappa: K \longrightarrow K^{\prime}, \lambda: L \longrightarrow$ $L^{\prime}, \alpha: L \longrightarrow K$ and $\alpha^{\prime}: L^{\prime} \longrightarrow K^{\prime}$ combinatorially commute and let $\kappa$ be combinatorial. Then

$$
\lambda\left(\alpha^{-1}(s t(a))\right) \subset \alpha^{\prime-1}(s t(\kappa(a))) \text { and } \kappa\left(s t((\alpha(b))) \subset \operatorname{st}\left(\left(\alpha^{\prime} \circ \lambda\right)(b)\right)\right.
$$

for every $a \in K$ and $b \in L$.

\section{Proof of Theorem 1.4}

By the Vietoris-Begle theorem the composition of $\mathbb{Z}_{(\mathcal{L})}$-acyclic maps of compacta is again a $\mathbb{Z}_{(\mathcal{L})}$-acyclic map. Then by virtue of Theorem 1.1 we may assume without loss of generality that $\operatorname{dim} X=n+1$. 
For every $i=1,2, \ldots$ we will construct spaces $L_{i}, W_{i}, S_{i}, M_{i}, K_{i}$, maps represented in the diagram

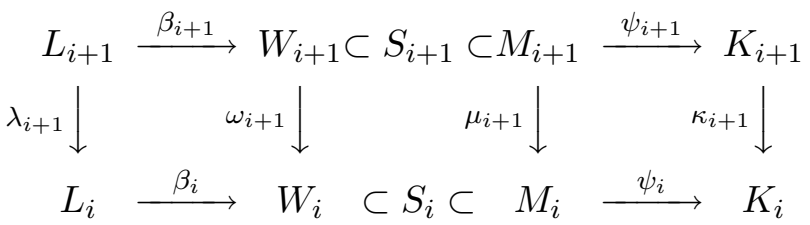

and some additional maps including $\phi_{i}: X \longrightarrow M_{i}$ and $f_{i}: X \longrightarrow K_{i}$ having the following properties.

Remark Since the list of properties (steps of the construction) is rather long we would like to outline a way to get the main idea of the construction omitting some details. The property (9) is the heart of the construction. The fastest way to get to (9) is to skip (8) and to omit all metric estimates in (1), (2), (6). Also note that the main purpose of (8), (11), (12) is to show that the maps $\kappa_{i+1}, \alpha_{i+1}, \lambda_{i+1}$ and $\alpha_{i}$ combinatorially commute where $\alpha_{i}=\psi_{i} \circ \beta_{i}: L_{i} \longrightarrow K_{i}$.

(1) $K_{i}$ is a finite simplicial complex of $\operatorname{dim} \leq n+1$. We fix a metric $d_{i}$ in $K_{i}$ such that the distance between any two points of $K_{i}$ lying in non-intersecting simplexes of $K_{i}$ is bigger than 1 . We also fix a metric in $X$.

(2) Define $\epsilon_{i}$ to be so small that for every $j \leq i$ and every $x, y \in K_{i}$ with $d_{i}(x, y)<\epsilon_{i}$ we have that $d_{j}\left(\kappa_{i}^{j}(x), \kappa_{i}^{j}(y)\right)<1 / 4^{i}$ where $\kappa_{i}^{i}=i d_{K_{i}}$ and $\kappa_{i}^{j}=$ $\kappa_{j+1} \circ \ldots \circ \kappa_{i}: K_{i} \longrightarrow K_{j}$ for $j<i$. By Proposition 2.1 we choose a CWcomplex $M_{i}$ and maps $\psi_{i}: M_{i} \longrightarrow K_{i}$ and $\phi_{i}: X \longrightarrow M_{i}$ such that the conclusions of Proposition 2.1] are satisfied with $K, M, \phi, \psi, f, \rho$ and $\epsilon$ replaced by $K_{i}, M_{i}, \phi_{i}, \psi_{i}, f_{i}, d_{i}$ and $\epsilon_{i} / 2$ respectively. Thus $M_{i}$ is a finite $\mathrm{CW}$-complex of $\operatorname{dim} \leq n+1$ admitting a compatible triangulation. We fix a compatible triangulation of $M_{i}$ and consider $M_{i}$ as both a CW-complex and a simplicial complex. Note that the CW-structure of $M_{i}$ is different from the simplicial structure of $M_{i}$ and therefore the cells of $M_{i}$ and simplexes of $M_{i}$ are not the same. The spaces $M_{i}$ are the only spaces in which we will consider simultaneously two structures.

(3) Let $W_{i}=$ the $n$-skeleton of $M_{i}$ with respect to the CW-structure of $M_{i}$.

Consider $W_{i}$ as a simplicial complex with a triangulation of $W_{i}$ to be a sufficiently small barycentric subdivision of the triangulation induced by the triangulation of $M_{i}$. We refer to this triangulation of $W_{i}$ considering simplexes of $W_{i}$. Thus the simplexes of $W_{i}$ and the simplexes of $M_{i}$ contained in $W_{i}$ are 
not the same. However each simplicial subcomplex of $M_{i}$ contained in $W_{i}$ will be also a subcomplex of $W_{i}$.

(4) Let $S_{i}=$ the $n$-skeleton of $M_{i}$ with respect to the triangulation of $M_{i}$.

Clearly $W_{i} \subset S_{i}$. Consider $S_{i}$ as a simplicial complex with the triangulation induced by the triangulation of $M_{i}$.

Let $\gamma_{i}: S_{i} \longrightarrow W_{i}$ be a natural retraction built by picking up in each $(n+1)$-cell of $M_{i}$ an interior point not belonging to $S_{i}$ and retracting the complement of the point inside the cell to the boundary of the cell. Then by (ii) of Proposition 2.1

$$
\gamma\left(\psi_{i}^{-1}(\Delta) \cap S_{i}\right) \subset \psi_{i}^{-1}(\Delta) \text { for every simplex } \Delta \text { of } K_{i} .
$$

Indeed, it is obvious if $\Delta$ is an $(n+1)$-simplex since then $\psi_{i}^{-1}(\Delta)$ is an $(n+1)$ cell of $M_{i}$. If $\Delta$ is of $\operatorname{dim} \leq n$ then $\psi_{i}^{-1}(\Delta) \subset W_{i}$ and we simply have $\gamma\left(\psi_{i}^{-1}(\Delta)\right)=\psi_{i}^{-1}(\Delta)$.

It is also easy to see that assuming that the triangulation of $M_{i}$ is sufficiently small $\gamma_{i}$ can be constructed such that for every $c \in W_{i}$ there exists a contractible subcomplex $N$ of $M_{i}$ contained in $W_{i}$ such that

$$
\gamma_{i}\left(s t\left(c, M_{i}\right) \cap S_{i}\right) \subset N \subset \psi_{i}^{-1}\left(s t\left(\psi_{i}(c)\right)\right)
$$

where $s t\left(c, M_{i}\right)$ is the star of $c$ with respect to the triangulation of $M_{i}$.

As we already noted in (3), $N$ is also a subcomplex of $W_{i}$.

(5) $L_{i}$ and $\beta_{i}: L_{i} \longrightarrow W_{i}$ are constructed as follows. $L_{i}$ is a simplicial complex of $\operatorname{dim} \leq n$ obtained from $W_{i}$ by replacing some $n$-simplexes of $W_{i}$ by $n$-dimensional $\mathcal{L}$-balls. Then $\beta_{i}$ is a projection of $L_{i}$ to $W_{i}$ such that $\beta_{i}$ takes the $\mathcal{L}$-balls to the corresponding replaced simplexes such that the nonboundary points of the $\mathcal{L}$-balls are sent to the interior of the replaced simplexes and $\beta_{i}$ is 1-to- 1 over all the simplexes of $W_{i}$ which are not replaced;

Let $i=1$. Set $\epsilon_{1}=1$. Let $K_{1}$ be any $(n+1)$-dimensional simplicial complex with any map $f_{1}: X \longrightarrow K_{1}$. Applying Proposition 2.1 define for $i=1$ all needed spaces and maps satisfying the relevant properties of (2-4). Define $L_{1}$ as $W_{1}$ with no simplexes replaced by $\mathcal{L}$-balls. We proceed from $i$ to $i+1$ as follows.

(6) Based on $\phi_{i}: X \longrightarrow M_{i}$ find a finite simplicial complex $K_{i+1}$ and maps $f_{i+1}: X \longrightarrow K_{i+1}$ and $\nu_{i+1}: K_{i+1} \longrightarrow M_{i}$ such that:

$d_{i}\left(\left(\psi_{i} \circ \nu_{i+1} \circ f_{i+1}\right)(x),\left(\psi_{i} \circ \phi_{i}\right)(x)\right)<\epsilon_{i} / 2$ for every $x \in X$ with $\epsilon_{i}$ defined in $(1)$; 
$f_{i+1}^{-1}(\Delta)$ is of diam $\leq 1 /(i+1)$ for every simplex $\Delta$ of $K_{i+1}$;

$\nu_{i+1}$ is a combinatorial map with respect to the triangulation of $M_{i}$.

Define $\kappa_{i+1}=\psi_{i} \circ \nu_{i+1}: K_{i+1} \longrightarrow K_{i}$ and suppose that the triangulation of $K_{i+1}$ is so small that

$\kappa_{i+1}^{j}(\Delta)$ is of diam $\leq 1 /(i+1)$ for every simplex $\Delta$ of $K_{i+1}$ and $j \leq i$.

(7) Let $M_{i+1}, S_{i+1}, W_{i+1}$ and the corresponding maps be defined as described in (2-4). Since $\nu_{i+1} \circ \psi_{i+1}: M_{i+1} \longrightarrow M_{i}$ is combinatorial with respect to the simplicial structures of $M_{i+1}$ and $M_{i}$, by (i) of Proposition 2.2 there is a simplicial approximation $\mu_{i+1}: M_{i+1} \longrightarrow M_{i}$ of $\nu_{i+1} \circ \psi_{i+1}$. Then $\mu_{i+1}\left(S_{i+1}\right) \subset$ $S_{i}$. By (ii) of Proposition 2.2 assume that that the triangulation of $W_{i+1}$ is chosen to be so small that the map $\left.\gamma_{i} \circ \mu_{i+1}\right|_{\ldots}: W_{i+1} \longrightarrow W_{i}$ admits a simplicial approximation $\omega_{i+1}: W_{i+1} \longrightarrow W_{i}$.

(8) Now we will verify a technical property which will be used later. Namely we will show that $\kappa_{i+1},\left.\psi_{i+1}\right|_{W_{i+1}}, \omega_{i+1}$ and $\left.\psi_{i}\right|_{W_{i}}$ combinatorially commute. This property is equivalent to

$$
\omega_{i+1}\left(\left(\kappa_{i+1} \circ \psi_{i+1}\right)^{-1}(\Delta) \cap W_{i+1}\right) \subset \psi_{i}^{-1}(\Delta) \text { for every simplex } \Delta \text { of } K_{i} .
$$

Let $\Delta$ be a simplex of $K_{i}$. Note that $\psi_{i}^{-1}(\Delta)$ is a subcomplex of $M_{i}$ with respect to both the $\mathrm{CW}$ and the simplicial structures of $M_{i}$. Then since $\mu_{i+1}$ is a simplicial approximation of $\nu_{i+1} \circ \psi_{i+1}$ and $\psi_{i}^{-1}(\Delta)$ is a simplicial subcomplex of $M_{i}$ we have

$$
\begin{aligned}
\mu_{i+1}\left(\left(\nu_{i+1} \circ \psi_{i+1}\right)^{-1}\left(\psi_{i}^{-1}(\Delta)\right)\right) & \subset\left(\nu_{i+1} \circ \psi_{i+1}\right)\left(\left(\nu_{i+1} \circ \psi_{i+1}\right)^{-1}\left(\psi_{i}^{-1}(\Delta)\right)\right) \\
& =\psi_{i}^{-1}(\Delta)
\end{aligned}
$$

and therefore

$$
\mu_{i+1}\left(\left(\nu_{i+1} \circ \psi_{i+1}\right)^{-1}\left(\psi_{i}^{-1}(\Delta)\right) \cap W_{i+1}\right) \subset \psi_{i}^{-1}(\Delta) \cap S_{i} .
$$

thus $\quad\left(\gamma_{i} \circ \mu_{i+1}\right)\left(\left(\nu_{i+1} \circ \psi_{i+1}\right)^{-1}\left(\psi_{i}^{-1}(\Delta)\right) \cap W_{i+1}\right) \subset \gamma_{i}\left(\psi_{i}^{-1}(\Delta) \cap S_{i}\right)$.

By (4) $\gamma_{i}\left(\psi_{i}^{-1}(\Delta) \cap S_{i}\right) \subset \psi_{i}^{-1}(\Delta) \cap W_{i}$ and since $\psi_{i}^{-1}(\Delta) \cap W_{i}$ is a subcomplex of $W_{i}$ and $\omega_{i+1}$ is a simplicial approximation of $\left.\gamma_{i} \circ \mu_{i+1}\right|_{\ldots}: W_{i+1} \longrightarrow W_{i}$ we get

$$
\omega_{i+1}\left(\left(\nu_{i+1} \circ \psi_{i+1}\right)^{-1}\left(\psi_{i}^{-1}(\Delta)\right) \cap W_{i+1}\right) \subset \psi_{i}^{-1}(\Delta) \cap W_{i} \subset \psi_{i}^{-1}(\Delta) .
$$

Recall that $\kappa_{i+1}=\psi_{i} \circ \nu_{i+1}$ and finally get that

$$
\omega_{i+1}\left(\left(\kappa_{i+1} \circ \psi_{i+1}\right)^{-1}(\Delta) \cap W_{i+1}\right) \subset \psi_{i}^{-1}(\Delta) \text { for every simplex } \Delta \text { of } K_{i} .
$$


(9) $L_{i+1}$ is constructed as follows. Let $C$ be an $(n+1)$-cell of $M_{i+1}$. By (ii) of Proposition 2.1 there is an $(n+1)$-simplex $\Delta$ of $K_{i+1}$ such that $C=\psi_{i+1}^{-1}(\Delta)$. By (iv) of Proposition [2.1 there is an $n$-simplex $\Delta_{C}$ of $W_{i+1}$ contained in $\partial C$ which is sent by $\psi_{i+1}$ to the interior of $\Delta$. Thus for every $(n+1)$-cell $C$ of $M_{i+1}$ we choose an $n$-simplex $\Delta_{C}$ of $W_{i+1}$ which consisits of free boundary points of $C$.

We say that an $n$-simplex $\Delta_{i+1}$ of $W_{i+1}$ is marked (for replacement) if $\Delta_{i+1}$ is not among the chosen simplexes $\Delta_{C}$ and $\Delta_{i+1}$ is mapped by $\omega_{i+1}$ onto an $n$-simplex $\Delta_{i}$ of $W_{i}$ such that $\Delta_{i}$ was replaced by an $n$-dimensional $\mathcal{L}$-ball $B_{\Delta_{i}}=\beta_{i}^{-1}\left(\Delta_{i}\right)$ while constructing $L_{i}$, see (5).

Replace each marked simplex $\Delta_{i+1}$ by an $n$-dimensional $\mathcal{L}$-ball $B_{\Delta_{i+1}}$ which is a copy of $B_{\Delta_{i}}$ attached by the simplicial map of the boundaries of $\Delta_{i+1}$ and $B_{\Delta_{i+1}}$ induced by $\omega_{i+1}$ and $\beta_{i}$. Such a replacement induces the natural map $\lambda_{\Delta_{i+1}}: B_{\Delta_{i+1}} \longrightarrow B_{\Delta_{i}}=\beta_{i}^{-1}\left(\Delta_{i}\right) \subset L_{i}$.

Let $C$ be an $(n+1)$-cell of $M_{i+1}$ and let $T_{C}$ be the union of all the simplexes of $W_{i+1}$ which are contained in $C$ and different from $\Delta_{C}$. Then after the replacement of all marked simplexes of $W_{i+1}$ we will obtain from $T_{C}$ an $n$ dimensional $\mathcal{L}$-ball $B_{C}$ and the map $\lambda_{C}: B_{C} \longrightarrow L_{i}$ induced by $\omega_{i+1}$ and $\beta_{i}$ for the simplexes of $T_{C}$ which were not replaced and by the maps $\lambda_{\Delta_{i+1}}$ for the replaced simplexes. Now replace $\Delta_{C}$ by an $\mathcal{L}$-ball $B_{\Delta_{C}}$ which is a copy of $B_{C}$ attached by the identity map of the boundaries and extend $\lambda_{C}$ over $B_{\Delta_{C}}$ first sending the points of $B_{\Delta_{C}}$ to the corresponding points of $B_{C}$ and from there to $L_{i}$ using the map $\lambda_{C}$. Thus we construct $L_{i+1}$ and the map $\lambda_{i+1}: L_{i+1} \longrightarrow L_{i}$ having the property that for every $(n+1)$-cell $C$ of $M_{i+1}$ the map

$$
\left.\lambda_{i+1}\right|_{\ldots}: \beta_{i+1}^{-1}(\partial C) \longrightarrow \lambda_{i+1}\left(\beta_{i+1}^{-1}(\partial C)\right)
$$

factors through an $n$-dimensional $\mathcal{L}$-ball (namely through $B_{C}$ ).

(10) Define $\alpha_{i}=\psi_{i} \circ \beta_{i}: L_{i} \longrightarrow K_{i}$.

Then the last property of (9) says that for every $(n+1)$-simplex $\Delta$ of $K_{i+1}$ the map $\left.\lambda_{i+1}\right|_{\ldots}: \alpha_{i+1}^{-1}(\Delta) \longrightarrow \lambda_{i+1}\left(\alpha_{i+1}^{-1}(\Delta)\right)$ factors through a $\mathbb{Z}_{(\mathcal{L})}$ - acyclic space.

(11) It follows from (9) that if $a \in \Delta_{C}$ then $\left(\beta_{i} \circ \lambda_{i+1}\right)\left(\beta_{i+1}^{-1}(a)\right) \subset \omega_{i+1}(\partial C)$ and if $a \in W_{i+1}$ does not belong to any of the simplexes $\Delta_{C}$ then simply $\beta_{i} \circ \lambda_{i+1}\left(\beta_{i+1}^{-1}(a)\right)=\omega_{i+1}(a)$.

Thus we obtain that for every simplex $\Delta$ of $K_{i+1}$

$$
\left(\beta_{i} \circ \lambda_{i+1}\right)\left(\beta_{i+1}^{-1}\left(\psi_{i+1}^{-1}(\Delta)\right)=\left(\beta_{i} \circ \lambda_{i+1}\right)\left(\alpha_{i+1}^{-1}(\Delta)\right) \subset \omega_{i+1}\left(\psi_{i+1}^{-1}(\Delta) \cap W_{i+1}\right) .\right.
$$


(12) Now we will show that the maps $\kappa_{i+1}, \alpha_{i+1}, \lambda_{i+1}$ and $\alpha_{i}$ combinatorially commute. Let $\Delta$ be a simplex of $K_{i}$. Then $\kappa_{i+1}^{-1}(\Delta)$ is a subcomplex of $K_{i}$ and by (11) and (8) we get that

$$
\begin{aligned}
\left(\beta_{i} \circ \lambda_{i+1}\right)\left(\left(\kappa_{i+1} \circ \alpha_{i+1}\right)^{-1}(\Delta)\right) & =\left(\beta_{i} \circ \lambda_{i+1}\right)\left(\alpha_{i+1}^{-1}\left(\kappa_{i+1}^{-1}(\Delta)\right)\right. \\
& \subset \omega_{i+1}\left(\psi_{i+1}^{-1}\left(\kappa_{i+1}^{-1}(\Delta)\right) \cap W_{i+1}\right) \subset \psi_{i}^{-1}(\Delta) .
\end{aligned}
$$

Thus we get that

$$
\left(\alpha_{i} \circ \lambda_{i+1}\right)\left(\left(\kappa_{i+1} \circ \alpha_{i+1}\right)^{-1}(\Delta)\right) \subset \Delta \text { for every simplex } \Delta \text { of } K_{i}
$$

and hence the maps $\kappa_{i+1}, \alpha_{i+1}, \lambda_{i+1}$ and $\alpha_{i}$ combinatorially commute.

Then by (iii) of Proposition 2.2 for every $a \in K_{i+1}$ and $b \in L_{i+1}$

$$
\lambda_{i+1}\left(\alpha_{i+1}^{-1}(s t(a))\right) \subset \alpha_{i}^{-1}\left(s t\left(\kappa_{i+1}(a)\right)\right)
$$

and

$$
\kappa_{i+1}\left(s t\left(\left(\alpha_{i+1}(b)\right)\right) \subset \operatorname{st}\left(\left(\alpha_{i} \circ \lambda_{i+1}\right)(b)\right) .\right.
$$

(13) Let $a \in K_{i+1}$ be such that $\kappa_{i+1}(a)$ is not contained in the interior of any of the $(n+1)$-simplexes of $K_{i}$. Then $\nu_{i+1}(a) \in W_{i}$. Let us show that the map $\left.\left.\lambda_{i+1}\right|_{\ldots}: \alpha_{i+1}^{-1}(s t(a))\right) \longrightarrow \alpha_{i}^{-1}\left(s t\left(\kappa_{i+1}(a)\right)\right)$ factors through a $\mathbb{Z}_{(\mathcal{L})^{-}}$acyclic space.

By (4) there is a contractible subcomplex $N$ of $W_{i}$ such that

$$
\gamma_{i}\left(s t\left(\nu_{i+1}(a)\right) \cap S_{i}\right) \subset N \subset \psi_{i}^{-1}\left(s t\left(\kappa_{i+1}(a)\right)\right.
$$

where $\operatorname{st}\left(\nu_{i+1}(a)\right)$ is the star of $\nu_{i+1}(a)$ with respect to the triangulation of $M_{i}$. Since $\nu_{i+1}$ is combinatorial with respect to the triangulation of $M_{i}$ we have

$$
\nu_{i+1}(s t(a)) \subset \operatorname{st}\left(\nu_{i+1}(a)\right)
$$

and therefore

$$
\nu_{i+1}\left(\psi_{i+1}\left(\psi_{i+1}^{-1}(s t(a))\right)\right)=\nu_{i+1}(s t(a)) \subset s t\left(\nu_{i+1}(a)\right) .
$$

Since $\mu_{i+1}$ is a simplicial approximation of $\nu_{i+1} \circ \psi_{i+1}$ and $\operatorname{st}\left(\nu_{i+1}(a)\right)$ is a simplicial subcomplex of $M_{i}$ we have

$$
\mu_{i+1}\left(\psi_{i+1}^{-1}(s t(a))\right) \subset \operatorname{st}\left(\nu_{i+1}(a)\right)
$$

and therefore

$$
\gamma_{i}\left(\mu_{i+1}\left(\psi_{i+1}^{-1}(s t(a)) \cap S_{i}\right) \subset \gamma_{i}\left(s t\left(\nu_{i+1}(a)\right) \cap S_{i}\right) \subset N .\right.
$$

Then since $N$ is a subcomplex of $W_{i}$ and $\omega_{i+1}$ is a simplicial approximation of $\left.\gamma_{i} \circ \mu_{i+1}\right|_{\ldots}: W_{i+1} \longrightarrow W_{i}$ we have

$$
\omega_{i+1}\left(\psi_{i+1}^{-1}(s t(a)) \cap W_{i}\right) \subset N
$$


and hence

$$
\omega_{i+1}\left(\psi_{i+1}^{-1}(s t(a)) \cap W_{i}\right) \subset N \subset \psi_{i}^{-1}\left(s t\left(\kappa_{i+1}(a)\right) .\right.
$$

By (11)

$$
\beta_{i}\left(\lambda_{i+1}\left(\alpha_{i+1}^{-1}(s t(a))\right)\right) \subset \omega_{i+1}\left(\psi_{i+1}^{-1}(s t(a)) \cap W_{i}\right)
$$

and therefore

$$
\lambda_{i+1}\left(\alpha_{i+1}^{-1}(s t(a))\right) \subset \beta_{i}^{-1}(N) \subset \beta^{-1}\left(\psi_{i}^{-1}\left(s t\left(\kappa_{i+1}(a)\right)\right)=\alpha_{i}^{-1}\left(s t\left(\kappa_{i+1}(a)\right)\right) .\right.
$$

By (5) the preimage under $\beta_{i}$ of every simplex of $W_{i}$ is $\mathbb{Z}_{(\mathcal{L})^{-}}$acyclic and therefore by the combinatorial Vietoris-Begle theorem $\beta_{i}^{-1}(N)$ is $\mathbb{Z}_{(\mathcal{L})}$ - acyclic.

Thus the map $\left.\left.\lambda_{i+1}\right|_{\ldots}: \alpha_{i+1}^{-1}(s t(a))\right) \longrightarrow \alpha_{i}^{-1}\left(s t\left(\kappa_{i+1}(a)\right)\right)$ factors through a $\mathbb{Z}_{(\mathcal{L})}$ - acyclic space.

(14) Let $a \in K_{i+2}$. By (12) consider the following maps

$$
\begin{aligned}
& \left.\lambda_{i+2}\right|_{\ldots}: \alpha_{i+2}^{-1}(s t(a)) \subset \alpha_{i+1}^{-1}\left(s t\left(\kappa_{i+2}(a)\right)\right) \text { and } \\
& \left.\lambda_{i+1}\right|_{\ldots}: \alpha_{i+1}^{-1}\left(\operatorname { s t } ( \kappa _ { i + 2 } ( a ) ) \subset \alpha _ { i } ^ { - 1 } \left(s t\left(\kappa_{i+1}\left(\kappa_{i+2}(a)\right)\right) .\right.\right.
\end{aligned}
$$

If $\kappa_{i+2}(a)$ is in the interior of an $(n+1)$-simplex $\Delta$ of $K_{i+1}$ then $s t\left(\kappa_{i+2}(a)\right)=$ $\Delta$ and by $\left.(10) \lambda_{i+2}\right|_{\ldots}$ factors through a $\mathbb{Z}_{(\mathcal{L})}$ - acyclic space.

If $\kappa_{i+2}(a)$ is not in the interior of any of the $(n+1)$-simplexes of $K_{i+1}$ then by (13) $\left.\lambda_{i+1}\right|_{\ldots}$ factors through a $\mathbb{Z}_{(\mathcal{L})}$ - acyclic space.

Thus we get that the map

$$
\left.\lambda_{i+1} \circ \lambda_{i+2}\right|_{\ldots}: \alpha_{i+2}^{-1}(s t(a)) \longrightarrow \alpha_{i}^{-1}\left(s t\left(\kappa_{i+1}\left(\kappa_{i+2}(a)\right)\right)\right.
$$

always factors through a $\mathbb{Z}_{(\mathcal{L})}$ - acyclic space.

(15) Define

$$
\begin{aligned}
& L=\lim _{\leftarrow}\left(L_{i}, \lambda_{i}\right) \text { and } K=\lim _{\leftarrow}\left(K_{i}, \kappa_{i}\right) \text { with the projections } \\
& \pi_{i}^{L}: L \longrightarrow L_{i} \text { and } \pi_{i}^{K}: K \longrightarrow K_{i} .
\end{aligned}
$$

It is clear that $\operatorname{dim} L \leq n$. For every $b=\left(b_{i}\right) \in L, b_{i} \in L_{i}$ define the set $\pi(b)=\cap\left\{\left(\pi_{i}^{K}\right)^{-1}\left(s t\left(\alpha_{i}\left(b_{i}\right)\right)\right): i=1,2,3 \ldots\right\} \subset K$. By (12) $\pi(b) \neq \emptyset$. Recall that by $(6) \operatorname{diam}\left(\kappa_{i}^{j}(\Delta)\right)<1 / i$ for every simplex $\Delta$ of $K_{i}$ and $j<i$. Then $\pi(b)$ is a singleton and by (12) one can check that the function

$\pi: L \longrightarrow K$ defined by $b \longrightarrow \pi(b)$ is continuous with $\pi^{-1}(a)=\lim _{\leftarrow}\left(\alpha_{i}^{-1}\left(s t\left(a_{i}\right)\right),\left.\lambda_{i}\right|_{\ldots}\right)$ for every $a=\left(a_{i}\right) \in K$. 
It is easy to see that for every simplex $\Delta$ of $K_{i}, \alpha_{i}^{-1}(\Delta) \neq \emptyset$. Then we have that for every $a \in K, \pi^{-1}(a)$ is not empty and by (14) we get that $\pi^{-1}(a)$ is $\mathbb{Z}_{(\mathcal{L})}$-acyclic.

Thus $\pi$ is a $\mathbb{Z}_{(\mathcal{L})}$-acyclic surjective map.

(16) Let $x \in X$ and $j \leq i$. Define $f_{i}^{j}=\kappa_{i}^{j} \circ f_{i}: X \longrightarrow K_{j}$.

Then $f_{i+1}^{i}=\kappa_{i+1} \circ f_{i+1}=\psi_{i} \circ \nu_{i+1} \circ f_{i+1}$ and by $(6) d_{i}\left(f_{i+1}(x),\left(\psi_{i} \circ \phi_{i}\right)(x)\right)<$ $\epsilon_{i} / 2$.

By (2) and (i) of Proposition 2.1 we have

$$
\left.d_{i}\left(f_{i}(x),\left(\psi_{i} \circ \phi_{i}\right)(x)\right)\right)<\epsilon_{i} / 2 \text { and hence } d_{i}\left(f_{i}(x), f_{i+1}^{i}(x)\right)<\epsilon_{i} .
$$

Then, since $f_{i+1}^{j}=\kappa_{i}^{j} \circ f_{i+1},(2)$ implies that $d_{j}\left(f_{i}^{j}(x), f_{i+1}^{j}(x)\right)<1 / 4^{i}$.

Now define $h_{j}: X \longrightarrow K_{j}$ as $h_{j}=\lim _{i \rightarrow \infty} f_{i}^{j}$.

Then $d_{j}\left(h_{j}(x), f_{j}(x)\right)<1 / 2$. Let $a=h_{j}(x)$ and let $y \in X$ be such that $h_{j}(y)=h_{j}(x)$. Then $d_{j}\left(f_{j}(y), f_{j}(x)\right)<1$ and hence by $(1) f_{j}(y) \in s t^{2}(a)$ where $s t^{2}(a)$ is the union of all simplexes of $K_{j}$ intersecting $s t(a)$.

Thus $h_{j}^{-1}\left(h_{j}(x)\right) \subset f_{j}^{-1}\left(s t^{2}(a)\right)$ and hence by $(6) h_{j}^{-1}\left(h_{j}(x)\right)$ is of diam $\leq 4 / j$.

It is clear that $h_{j}=\kappa_{j+1} \circ h_{j+1}$. Then we have that $h: X \longrightarrow K$ defined by $h(x)=\left(h_{j}(x)\right) \in K$ is an embedding of $X$ in $K$.

Identify $X$ with $h(X)$. Then $r=\left.\pi\right|_{\ldots}: Z=\pi^{-1}(X) \longrightarrow X$ is the required resolution.

Remark If $n \geq 3$ then in (9) $B_{C}$ is simply connected and one can use the Hurewicz theorem to replace each simplex $\Delta_{C}$ by an $n$-cell attached to the boundary by a map of degree not divisible by the elements of $\mathcal{L}$ such that there is a map from the replacing cell to $B_{C}$. In our construction we used an exact copy of $B_{C}$ for replacing $\Delta_{C}$ in order to guarantee that such a map exists for the non-simply connected case $n=2$. 


\section{References}

[1] R. D. Edwards, A theorem and a question related to cohomological dimension and cell-like maps, Notices of the AMS, 25(1978), A-259.

[2] A. N. Dranishnikov, On homological dimension modulo $p$. (Russian) Mat. Sb. (N.S.) 132(174) (1987), no. 3, 420-433, 446; translation in Math. USSR-Sb. 60 (1988), no. 2, 413-425. MathReview

[3] A. N. Dranishnikov, Extension of mappings into CW-complexes. (Russian) Mat. Sb. 182 (1991), no. 9, 1300-1310; translation in Math. USSR-Sb. 74 (1993), no. 1, 47-56. MathReview

[4] A. N. Dranishnikov, Rational homology manifolds and rational resolutions. Special issue in memory of B. J. Ball. Topology Appl. 94 (1999), no. 1-3, 75-86. MathReview

[5] A. Dranishnikov, Cohomological dimension theory of compact metric spaces, Topology Atlas Invited Contributions, http://at.yorku.ca/topology/taic.htm

[6] A. Koyama and K. Yokoi, Cohomological dimension and acyclic resolutions. In memory of T. Benny Rushing. Topology Appl. 120 (2002), no. 1-2, 175-204. MathReview

[7] M. Levin, Acyclic resolutions for arbitrary groups, Israel J. Math., 135(2003), 193-204. MathReview

[8] M. Levin, Universal acyclic resolutions for finitely generated coefficient groups, Topology Appl., 135(2004), 101-109. MathReview

[9] M. Levin, Universal acyclic resolutions for arbitrary coefficient groups, Fund. Math., 178(2003), 159-169. MathReview

[10] J. Walsh, Dimension, cohomological dimension, and cell-like mappings. Shape theory and geometric topology (Dubrovnik, 1981), pp. 105-118, Lecture Notes in Math., 870, Springer, Berlin-New York, 1981. MathReview

Department of Mathematics, Ben Gurion University of the Negev

P.O.B. 653, Be'er Sheva 84105, ISRAEL

Email: mlevine@math.bgu.ac.il

Received: 17 March 2004 Revised: 22 March 2005 